\title{
Limits of Spatial Vision in Sumatran Orangutans (Pongo abelii)
}

\author{
Laura C. Adams, Frances Wilkinson, and Suzanne E. MacDonald*
}

York University, Toronto, Canada

*Corresponding author (Email: suzmac@yorku.ca)

Citation - Adams, L., Wilkinson, F., \& MacDonald, S. E. (2017). Limits of spatial vision in Sumatran orangutans (Pongo abelii). Animal Behavior and Cognition, 4(3), 204 - 222. https://doi.org/10.26451/abc.04.03.02.2017

\begin{abstract}
Although research with animals is often done under the assumption that visual abilities are similar across species, the visual ability of most animals, including orangutans, has not been experimentally evaluated. In this study we assessed the contrast sensitivity function (CSF) of two female zoo-housed Sumatran orangutans (Pongo abelii) aged 20 and 26 years old. Orangutans were rewarded for selecting vertical or horizontal square wave gratings at the correct orientation. Results showed a CSF similar in shape and position to that of human adults, although with lower contrast sensitivity. These lower values may be due to testing constraints or may be due to species differences. These data have implications for research on orangutan cognition, hominid evolution, and have practical implications for captive and wild management of this endangered species.
\end{abstract}

Keywords - Visual perception, Contrast sensitivity, Spatial frequency, Sensory ecology, Orangutan

Primate vision shows similarities and differences across taxonomic and geographic divides as well as across habitat, life history, and ecological niche (Matsuno \& Fujita, 2009; Veilleux \& Kirk, 2014). For example, there are differences in color vision and visual acuity across nocturnal, diurnal, and cathemeral primates (Jacobs, 1977, 1996; Kirk, 2004; Matsui, Go, \& Niimura, 2010; Ordy \& Samorajski, 1968; Veilleux \& Kirk, 2014). Eye morphology and visual system anatomy and physiology are similar within Old World monkeys and apes, but differences exist between these species and New World primates (Chan \& Grünert, 1998; Waitt \& Buchanan-Smith, 2006), and between haplorhine and strepsirrhine suborders of primates (Kirk, 2004; Veilleux \& Kirk, 2009). Our closest relatives are the other great apes, yet there is limited information about either behavioral or physiological aspects of vision in these species. The purpose of the present study was to examine basic spatial vision limits in orangutans. Orangutans are often assumed to have identical vision to humans, and this assumption underlies cognitive testing with the species. However, there are few published reports of orangutan visual ability, and no reports of the orangutan contrast sensitivity function.

\section{Orangutan Life History}

Orangutans are an important species to study to understand great ape evolution because this species had an earlier phylogenetic split and geographic isolation in Asia from the African great apes. Evaluations of human phylogenetic relationships typically cite a common ancestor with chimpanzees (Pan troglodytes) 6 million years ago (MYA), and a common ancestor with orangutans 14 MYA (Goodman et al., 1998). Genetic comparisons estimate the split between two extant species of orangutan, Sumatran (Pongo abelii) and Bornean (Pongo pygmaeus), to be 330 thousand years ago (Mailund, Dutheil, Hobolth, Lunter, \& Schierup, 2011) or even earlier (Becquet \& Przeworski, 2007).

Orangutans also provide a valuable comparison to other great apes due to differences in ecological 
niche. They spend more time in the tree canopy in comparison to other great apes and live predominantly in lowland areas, including peat swamps (Rijksen, 1978; van Schaik, 2004). This habitat is characterized by low-level light conditions, with mottled light that has been filtered through the leaves of the forest canopy. In these conditions orangutans must find food, which can be difficult to identify and requires extensive travel to locate (Rijksen, 1978). The majority of the Sumatran orangutan diet is fruit, but they also consume bark, leaves, pith, flowers, sap, roots, seeds, honey, fungi, mineral-rich soil, eggs, invertebrates, and meat, and some of these foods require precise manipulation and tool use (Hardus et al., 2012; Rijksen, 1978; van Schaik, 2004; Wich et al., 2004). Like other primates, vision is their dominant sense, and thus relied on by orangutans to locate and identify these foods (Gilad, Wiebe, Przeworski, Lancet, \& Pääbo, 2004; Matsuno \& Fujita, 2009; Schrauf \& Call, 2009). Vision is also crucial for arboreal travel. Because orangutans are large and heavy in comparison to other arboreal animals, accurate estimation of distance and quality of branch support is essential in preventing potentially fatal falls (Schmitt, 2010; van Schaik, 2004). Orangutan vision is also necessary for other aspects of daily life that require perception of fine detail and subtle contrast differences including tool use and viewing social partners (Rijksen, 1978; van Schaik, 1999, 2004), which they often perform in low light and in the forest canopy.

\section{The Orangutan Eye and Brain}

There are no reported empirical tests of orangutan vision other than a study showing that a Bornean orangutan could discriminate grey paper in comparison to red, blue, green, and yellow (Tigges, 1963). However, there have been some preliminary anatomical measurements of orangutan eyes and visual cortex. Hotta (1905) reported detailed measurements of the globe and retina of orangutans, along with chimpanzees, gorillas (Gorilla sp.) and gibbons (Hylobates sp.), and confirmed the presence of a fovea in all these species. In more recent studies, Kirk $(2004,2006)$ reported comparable measurements in one Bornean orangutan: mean transverse eye diameter of $22.8 \mathrm{~mm}$, axial eye diameter of $22.6 \mathrm{~mm}$, transverse corneal diameter of $11.6 \mathrm{~mm}$, and an orbital aperture diameter of $36.1 \mathrm{~mm}$, values similar to those of a chimpanzee (23.0, 21.8, 10.2, and $30.8 \mathrm{~mm}$ respectively). Montiani-Ferreira et al. (2010) noted that, while current knowledge about orangutan ocular anatomy and physiology is limited, orangutan eye physiology is similar enough to human eye physiology that human doctors were able to perform successful cataract surgery on a captive Bornean orangutan. Post mortem anatomical examination of the connections of short wavelength-sensitive (SWS) cones and horizontal cells in the retinas of a Bornean orangutan and a chimpanzee revealed similarity to humans in terms of the pattern of $\mathrm{H} 1$ versus $\mathrm{H} 2$ connections (Chan \& Grünert, 1998). However, no other information about orangutan retinal organization is available.

An excellent general description of the orangutan brain compared to those of other great apes and humans is provided by Zilles and Rehkämper (1988). They concluded that, in terms of gross features and a scale of encephalization, the orangutan is most similar to the gorilla with both these species showing lower indices of telencephalic and cortical development than gibbon, chimpanzee or human. In the only recent studies we are aware of in which orangutan visual cortex was investigated (Preuss, Qi, \& Kaas 1999), the dense band of cytochrome oxidase staining seen in layer 4A of Old and New World Monkeys was absent in human, chimpanzee and orangutan. On the other hand, a clear difference was seen between human V1 tissue and that of both the other great apes and the monkeys in terms of the pattern of presumed M-pathway targets in Layer 4A as revealed by immunological staining (Preuss et al., 1999, Preuss \& Coleman, 2002). Finally, a recent study using post-mortem MRI measurements reported LGN and V1 volumes for three orangutans along with other great apes (De Sousa et al., 2010), and a second study of sectioned material from the same animals made cytoarchitectural comparisons of several cortical areas, including V1 across all the great apes (Semendeferi et al., 2011). Beyond these studies nothing is known of orangutan central visual pathways. 


\section{Limits of Primate Visual Ability}

Visual systems can be characterized in terms of the limits of their ability to encode spatial, temporal, and wavelength information about the changing visual environment. Extensive laboratory-based behavioral and physiological studies exist that compare human visual systems to the most frequently studied nonhuman primate species, macaques, looking at all three of these properties but information is much more limited for the full range of primates, and particularly for nonhuman great apes. Spatial vision has been most widely investigated, followed by color vision (for a review see Matsuno \& Fujita, 2009). Data on temporal processing (motion and flicker sensitivity) is largely lacking in nonhuman primate species outside macaques (Macacca fascicularis) (O’Keefe \& Movshon, 1998) and chimpanzees (Matsuno \& Tomonaga, 2006b, 2008), yet is likely to be a critical sensitivity for skills such as arboreal travel.

Spatial vision refers to the ability to distinguish changes based on luminance variation across the visual field and to use these changes as a basis for constructing a representation of the layout and identity of objects in the visual scene. The contrast threshold refers to the minimum detectable difference in luminance between adjacent spatial regions. It is typically measured in the laboratory using a periodic pattern of parallel lines (a square or sine wave grating) and systematically reducing the luminance difference between the lighter (initially white) and darker (initially black) bars until they are an indistinguishable middle grey. Contrast sensitivity (CS) is the reciprocal of contrast threshold. However, CS varies as a function of the width of the bars and the viewing distance, which together determine the angle subtended at the eye by the stimulus (visual angle) and the size of the resulting retinal image. For periodic stimuli such as gratings, this measure is expressed as spatial frequency (SF) or the number of cycles of grating making up one degree of visual angle (c/deg). The contrast sensitivity function (CSF) comprises the CS limit across the range of visible spatial frequencies. Under high luminance and optimal conditions, human and macaque CSFs show peak sensitivity between 2 and $5 \mathrm{c} / \mathrm{deg}$ with CS decreasing at lower and higher SF (Arundale, 1978; Boothe, Kiorpes, Williams, \& Teller, 1988; Campbell \& Robson, 1968; De Valois, Morgan, \& Snodderly, 1974; Ross, Clarke, \& Bron, 1985; Williams, Boothe, Kiorpes, \& Teller, 1981; see Table 1 for a summary of CSF findings in primates). Sensitivity values at the peak range from $150-300$ (contrasts of $0.3-0.6 \%$ ) under optimal test conditions in these studies. The only study of CS in a non-human great ape (Matsuno \& Tomonaga, 2006a) reported CSFs similar in shape although slightly lower in absolute sensitivity in four chimpanzees compared to the one human observer tested in the same apparatus. However, the location of the peak and the value of peak CS in this study were considerably lower than most values reported in the literature for human subjects (Arundale, 1978; Ross et al., 1985).

The highest frequency (finest lines) at which a grating can be detected at maximum (100\%) contrast is the spatial resolution or visual acuity limit. This limit, when extrapolated from a measured CSF, corresponds quite closely to acuity measures obtained by more traditional means, namely reducing the SF/size of high contrast grating or other pattern until the stripes or gap cease to be detectable (Jacobs, 1977; Langston, Casagrande, \& Fox, 1986). When contrast is maximized, humans, pigtailed macaques (Macaque nemestrina), and long-tailed macaques (Macaca fascicularis) can distinguish over $30 \mathrm{c} / \mathrm{deg}$, with acuity estimates averaging 35 - 50 c/deg (Arundale, 1978; Boothe et al., 1988; De Valois et al., 1974; Ross et al., 1985; Williams et al., 1981). 
Table 1

The Contrast Sensitivity Function in Primates

\begin{tabular}{|c|c|c|c|c|c|c|}
\hline Authors & $\begin{array}{l}\text { Species }(\mathrm{N}, \text { age }) ; \\
\text { [Human comparison] }\end{array}$ & Activity & Method & Luminance & $\begin{array}{l}\text { Peak SF; CS } \\
\text { [Human] }\end{array}$ & $\begin{array}{l}\text { Extrapolated SF cutoff } \\
(\mathrm{CS}=1)\end{array}$ \\
\hline Langston et al., 1986 & $\begin{array}{l}\text { Galago Otolemur crassicaudatus } \\
(N=2 \text {, adult })\end{array}$ & Nocturnal & MOCS & $22 \mathrm{~cd} / \mathrm{m}^{2}$ & 1c/deg; 100 & $2.6 \& 4.4 \mathrm{c} / \mathrm{deg}$ \\
\hline Jacobs, 1977 & $\begin{array}{l}\text { Owl monkey Aotus trivirgatus } \\
(N=2 \text {, adult }) \\
{[\mathrm{H}(N=1, \text { adult })]}\end{array}$ & Nocturnal & MOCS & $11.4 \mathrm{~cd} / \mathrm{m}^{2}$ & $1.5-2 \mathrm{c} / \mathrm{deg} ; \leq 100$ & $\leq 10 \mathrm{c} / \mathrm{deg}$ \\
\hline Merigan, 1976 & $\begin{array}{l}\text { Squirrel monkey Saimiri sciureus } \\
(N=3 \text {, adult }) \\
{[\mathrm{H}(N=2, \text { adult })]}\end{array}$ & Diurnal & MOCS & $3 \mathrm{~cd} / \mathrm{m}^{2}$ & $\begin{array}{l}2-5 \mathrm{c} / \mathrm{deg} ; 95- \\
120 ;[\mathrm{H}: 200]\end{array}$ & $\begin{array}{l}17-35 \mathrm{c} / \mathrm{deg} \\
{[\mathrm{H}: 35 \mathrm{c} / \mathrm{deg}]}\end{array}$ \\
\hline De Valois et al., 1974 & $\begin{array}{l}\text { Macaque nemestrina; fasciculari } \\
(N=2,1, \text { adolescent }) ; \\
{[\mathrm{H}(N=5,15-25 \text { years })]}\end{array}$ & Diurnal & MOCS & $17 \mathrm{~cd} / \mathrm{m}^{2}$ & $\begin{array}{l}3-5 \text { c/deg; } 100 \\
{[\mathrm{H}: 200]}\end{array}$ & $40-50 \mathrm{c} / \mathrm{deg}$ \\
\hline Williams et al., 1981 & $\begin{array}{l}\text { Macaque nemestrina } \\
(N=4,5-18 \text { months }) \\
{[\mathrm{H}(N=2, \text { adult })]}\end{array}$ & Diurnal & MOCS & $27 \mathrm{~cd} / \mathrm{m}^{2}$ & $\begin{array}{l}3-6 \mathrm{c} / \mathrm{deg} ; 150 \\
{[\mathrm{H}: 300]}\end{array}$ & $40-50 \mathrm{c} / \mathrm{deg}$ \\
\hline Boothe et al., 1988 & $\begin{array}{l}\text { Macaque nemestrina } \\
(N=7,1-12 \text { months })\end{array}$ & Diurnal & MOCS & $27 \mathrm{~cd} / \mathrm{m}^{2}$ & $\begin{array}{l}3-6 \mathrm{c} / \mathrm{deg} ; 100(1 \\
\text { year }) * \text { variable }\end{array}$ & $30-50 \mathrm{c} / \mathrm{deg}(1$ year $)$ \\
\hline Campbell \& Robson, 1968 & Human study $(N=2)$ [adult] & Diurnal & $\begin{array}{l}\text { Method of } \\
\text { adjustment }\end{array}$ & $\begin{array}{l}500 \mathrm{~cd} / \mathrm{m}^{2}, \& \\
0.5 \mathrm{~cd} / \mathrm{m}^{2}\end{array}$ & $4 \mathrm{c} / \mathrm{deg} ; 300+$ & $\sim 45 \mathrm{c} / \mathrm{deg}$ \\
\hline Matsuno \& Tomonaga, 2006a & $\begin{array}{l}\text { Chimpanzee } \text { Pan troglodytes } \\
(N=4,21-28 \text { years }) \\
{[\mathrm{H}(N=1, \text { adult? })]}\end{array}$ & Diurnal & $\begin{array}{l}\text { PEST (1-up-1- } \\
\text { down) }\end{array}$ & $30 \mathrm{~cd} / \mathrm{m}^{2}$ & $1-2 \mathrm{c} / \mathrm{deg} ; \leq 100$ & not specified \\
\hline Current article & $\begin{array}{l}\text { Orangutan Pongo abelii } \\
(N=2,20,26 \mathrm{yr}) ; \\
{[\mathrm{H}(N=3, \text { adult })]}\end{array}$ & Diurnal & $\begin{array}{l}\text { Staircase (2- } \\
\text { down-1-up) }\end{array}$ & $19-22 \mathrm{~cd} / \mathrm{m}^{2}$ & $2-3 \mathrm{c} / \mathrm{deg}$ & $\begin{array}{l}13-20 \mathrm{c} / \mathrm{deg} ; \\
{[\mathrm{H}: 30-45+\mathrm{c} / \mathrm{deg}]}\end{array}$ \\
\hline
\end{tabular}

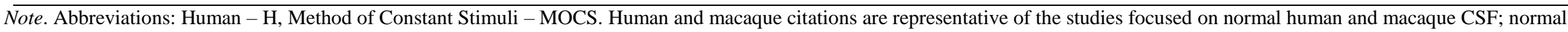
control CSFs are also reported in numerous clinical studies. 
Visual acuity rather than contrast sensitivity has frequently been used as a basis for describing the visual ability of a species and for cross-species comparisons. For example, Veilleux and Kirk (2014) have described the relationship between acuity and eye size and shape, and related this to habitat considerations. However, practical as well as theoretical considerations led us to choose CS as our measure. Practically, in order to measure acuity it is necessary to have available a range of stimuli that tightly bracket the SF range in which the threshold is likely to fall. Based on overall anatomical, ecological, and behavioral similarities to the other great apes, we expected that the visual acuity of the orangutan might be very high $(35-50$ c/deg) because values in this range have been reported in chimpanzees (Bard, Street, McCrary, \& Boothe, 1995; Spence, 1934; Weinstein \& Grether, 1940), as well as in humans, as discussed above. Producing adequate acuity stimuli for human subjects is not difficult because very long viewing distances can be used; clinical eye charts, for example, are typically used at a viewing distance of $20 \mathrm{ft}$. However, it is difficult to train non-human species on tasks without close spatial contiguity between stimulus, response, and reward (see Cowey \& Ellis, 1967, for discussion of this issue). For animals with much lower visual acuity $(<8$ c/deg), this can be successfully achieved using the Lashley jumping stand, in which the animal jumps toward a visual pattern (lemur Eulemur macaco flavifrons: Veilleux \& Kirk, 2009; gerbil Meriones unguiculatus: Wilkinson, 1984), or as in the Mitchell adaptation for cats (Felis catus), jumping down onto a patterned surface (Mitchell, Giffin, Wilkinson, Anderson, \& Smith, 1976). However, it is extremely difficult to produce, either by digital printing or electronically, a grating pattern as fine as $50 \mathrm{c} / \mathrm{deg}$ when within reach of the subject. In a highly constrained laboratory situation, it is possible to create the impression of such contiguity by having the animal look through a mask or through push-panel windows toward stimuli placed at some distance at the end of tunnels, which mask out all other visual stimulation. However, this was not feasible in a zoo setting. To give the reader an understanding of the fine scale of patterns needed to reach human or macaque acuity limits at close range, imagine painting a set of 50 pairs of precisely equally wide black and white lines across the width of your fingernail, and then hold the finger at arm's length from your eye. Such a pattern is exceptionally difficult to produce and impossible to calibrate. So practically, it was more feasible to measure contrast thresholds using lower frequency $(2-15$ c/deg) printed patterns that the animals could physically contact.

There are also theoretical reasons to measure CS rather than acuity. The CSF is now understood to reflect the envelope of sensitivities of multiple spatial channels within the visual pathway, carrying information at several different spatial scales (Campbell \& Robson, 1968; Lesmes, Lu, Baek, \& Albright, 2010; Wilson \& Giese, 1977) and thus, gives a more complete picture of spatial sensitivity. CSFs have been measured in a wide range of species including cats, pigeons, several rodents and several primates (see Uhlrich, Essock, \& Lehmkuhle, 1981, for review, and Table 1 for primates), and the general form of the CSF is similar across species, if normalized for maximum sensitivity and scaled in terms of distance from the peak frequency in octaves (factors of 2) (Uhlrich et al., 1981). Because acuity is simply the limit of the CSF - the SF beyond which even $100 \%$ contrast patterns cannot be resolved, it can be extrapolated from the CSF provided enough values are tested to adequately determine the shape of the function. Finally, it has been argued that the CSF has more relevance to daily life than acuity (Owsley \& Sloane, 1987), and this is likely also true for orangutans. Very few meaningful stimuli in the animal's natural world occur at extremely high SF and 100\% contrast. Most stimuli of importance in daily life, for example, the facial expression of a conspecific or a branch while brachiating, entail lower contrast and spatial frequencies well below the acuity limit.

\section{Purpose and Approach of the Present Study}

When the possibility of evaluating spatial vision in Sumatran orangutans at the Toronto zoo emerged, there were both theoretical and practical reasons to do so. Visual stimuli have been used in cognitive experiments with orangutans to assess abilities such as memory (Swartz, Himmanen, \& Shumaker, 2007), numerosity (Vonk, 2014), categorization (Marsh \& MacDonald, 2008; Vonk \& MacDonald, 2004), spatial cognition (Marsh, Spetch, \& MacDonald, 2011), and language and symbol use 
(Miles, 1990), yet there was no basis other than assumed similarity to human visual ability to guide stimulus design. Secondly, assessment of orangutan vision has practical application in regards to zoo management and veterinary baselines, especially important for maintaining this Critically Endangered species (Singleton, Wich, Nowak, \& Usher, 2016). And more theoretically, evaluating continuity and discontinuity in abilities across great apes, other primates, and other animals, can inform our evolutionary understanding of ancestral Hominoidea and primates in general.

The limitations of the zoo environment precluded the use of the highly controlled stimulus presentation sources used in earlier CSF and acuity studies of humans and other diurnal primates. In any case, the technologies generally used in the past (analogue oscilloscope displays, Ives visual acuity tester) are now obsolete and hard to obtain, and modern digital displays present other challenges in terms of luminance calibration. Instead, we turned to methods developed in laboratory studies of preverbal children and animals using hard-copy stimuli. Whereas many CS studies have been based on the discrimination of grating patterns from a matched homogeneous grey, it is difficult to obtain perfect luminance matches with printed stimuli. Instead, we chose to use the more difficult discrimination between vertical and horizontal grating patterns, which allowed us to use the same physical stimuli as either vertical or horizontal simply by rotating them. Because of the need for stimulus-response spatial contiguity, we used a set-up in which the orangutans could physically touch the stimulus. Our goal was a modest one - to measure contrast thresholds at several spatial frequencies that would include values close to the peak of sensitivity, and values at frequencies that we anticipated might fall on the declining portion of the CSF, thus allowing us to plot the shape of the function. This, we hoped, would allow us to extrapolate an acuity estimate even though it was beyond our capabilities to create sufficiently fine grating patterns to capture the resolution limit of the system. Despite limitations encountered in producing extremely low contrast gratings, we were successful in achieving contrast threshold measures in two orangutans, which we compare to human participants, and to existing chimpanzee data (Matsuno \& Tomonaga, 2006a).

\section{Method}

\section{Subjects}

Two of six Sumatran orangutans (Pongo abelii) residing at the Toronto Zoo participated in the study. All six orangutans were screened; however only two reached the initial training criterion within three months of training. The two subjects were both captive-born adult female orangutans. Sekali was 20 years old and Ramai was 26 years old. The study was conducted in the context of the orangutans' regular schedule and environment, and participation was completely voluntary. Orangutans had ad libitum access to water and their diet was not altered. Data were collected in the off-exhibit holding enclosures where there were climbing structures, bedding materials, and items for behavioral enrichment such as buckets and toys. The orangutans could also see and hear their conspecifics, so motivation and participation fluctuated due to these distractions. This study complied with the ethics and research protocols of York University and Toronto Zoo, and the laws of Canada.

\section{Apparatus and Stimuli}

Stimuli were presented to the orangutans on a wooden display unit measuring 57 x 45 x $42 \mathrm{~cm}$. The display unit had two podiums (left and right), which held the stimuli $17 \mathrm{~cm}$ apart (Figure 1). The unit also had a movable panel to obscure the orangutan's view of the stimuli in between trials. Once the orangutan was sitting, the unit was placed so that the stimuli were at a distance of $57 \mathrm{~cm}$ from the eyes of the orangutan. This distance was verified frequently, and remained constant because the orangutans held their faces against the mesh of the enclosure. If the orangutan moved or changed position, the location of the apparatus and distance were readjusted and confirmed. The orangutans used a wooden dowel to select the stimulus. 


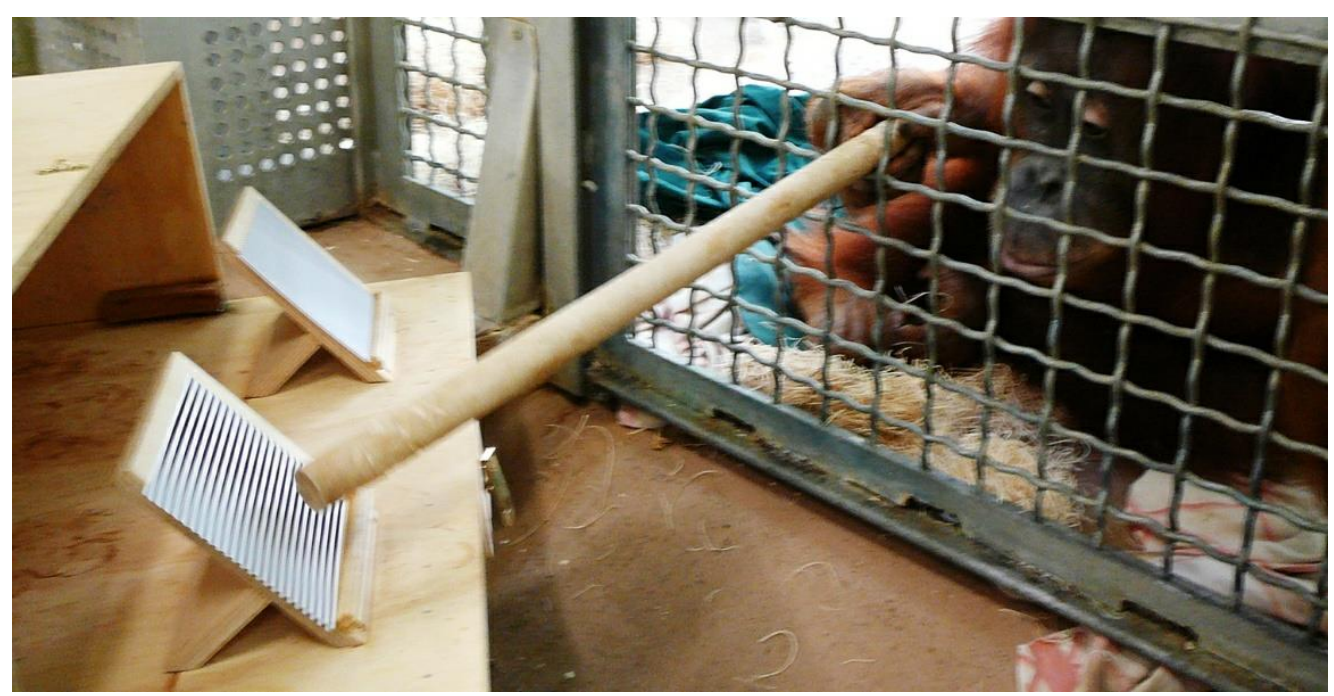

Figure 1. Stimulus display unit. During testing the stimuli were centered on the podiums with one oriented horizontally and one oriented vertically.

We created square wave grating stimuli using Adobe Photoshop CS5 computer software, printed them on non-glossy photo paper using a Canon Pixma MG6220 ink jet printer, and cut them into 12 x 12 $\mathrm{cm}$ squares. At a distance of $57 \mathrm{~cm}$ from the orangutan's eyes, one centimeter is equivalent to one degree of visual angle, so each stimulus subtended $12 \times 12$ degrees of visual angle. The target and comparison stimuli did not differ; they were taken from the same set of printed gratings. On each trial, two stimuli were selected from the set and placed on the display unit with one oriented vertically and the other horizontally. We printed a new set of stimuli for each testing day to ensure that there were no identifying marks on the stimuli. The orientation and/or the position of the stimulus cards was shuffled between trials so that across trials individual cards appeared as both target and comparison, and on both left and right podiums. Stimuli were replaced immediately if any identifying marks occurred.

The stimuli were printed for 2, 4, 10, and $15 \mathrm{c} / \mathrm{deg}$ when viewed at a distance of $57 \mathrm{~cm}$. A set of lower frequency stimuli $(0.5 \mathrm{c} / \mathrm{deg})$ was produced for training purposes. We printed each of the four spatial frequencies at seven levels of contrast. Contrast values ranged from the highest to the lowest contrast that could be produced by the printer and computer software. The actual contrast values of the stimuli were obtained from luminance measurements made with a Minolta LS-100 photometer (Table 2). Maximum and minimum luminance values were used to calculate the Michelson contrast as follows:

$$
\frac{L_{\text {max }}-L_{\text {min }}}{L_{\text {max }}+L_{\text {min }}}
$$

The photometer was used to measure the Michelson contrast in multiple locations within the testing area over two days and found reliability in the values across locations and days. Contrast sensitivity (CS) was calculated as the reciprocal of the threshold ( $C S=1 /$ Contrast Threshold $)$. The overall average luminance of all stimuli was similar, ranging between $19-22 \mathrm{~cd} / \mathrm{m}^{2}$ across stimuli. 
Table 2

Average Michelson Contrast Values for Stimuli

\begin{tabular}{ccccc}
\hline $\begin{array}{c}\text { Staircase } \\
\text { Step }\end{array}$ & Michelson Contrast & SD & Percent Contrast & Contrast Sensitivity \\
\hline 1 & 0.93 & .00 & 93 & 1.08 \\
2 & 0.56 & .02 & 56 & 1.79 \\
3 & 0.44 & .02 & 44 & 2.27 \\
4 & 0.31 & .02 & 31 & 3.23 \\
5 & 0.15 & .03 & 15 & 6.67 \\
6 & 0.09 & .01 & 9 & 11.11 \\
7 & 0.03 & .03 & 3 & 33.33 \\
\hline
\end{tabular}

\section{Procedure}

The orangutans were randomly assigned to select a target, with Sekali trained to select vertical and Ramai trained to select horizontal lines. On each testing trial two gratings were displayed, one oriented vertically and one oriented horizontally, matched in SF and contrast. Left and right locations of the target stimulus were in a predetermined order that was randomized and limited to three successive trials at a location. A research assistant loaded the two stimuli onto the two podiums of the display unit so that the experimenter was blind to the location of the target stimulus. On each trial the experimenter removed a panel to reveal the stimuli to the orangutan, but the stimuli were out of view of the experimenter. Once the orangutan had made a selection by touching a podium with the dowel, the experimenter verified whether the orangutan had selected the target by leaning forward and peeking over the display unit to see the stimuli. If the target was correctly selected, the orangutan was reinforced immediately with either verbal praise: "Good job." for Sekali or a whistle for Ramai. The orangutans also received a food reward: small pieces of fresh or dried fruit, nut, or candy. If the orangutan selected the comparison stimulus instead of the target, the experimenter tapped on the correct stimulus with a finger and said: "This one." for Sekali or remained silent for Ramai. The experimenter then closed the display unit and did not offer a food reward. Selection was defined as the first stimulus that the orangutan touched with the dowel. Any unclear selections, such as touching the space between the podiums, were recorded as incorrect trials.

The initial training stimulus was a highly visible grating ( $0.5 \mathrm{c} / \mathrm{deg}$ and Michelson contrast of 0.93$)$. Early training consisted of up to 60 trials per day to maximize learning given the constraints of the zoo schedule. Discrimination testing began on the first day that the orangutan selected the target stimulus on 17 out of the first 20 trials. Discrimination testing consisted of 20 trials per day on three consecutive days. Once the orangutans reached the criterion of 17 out of 20 correct selections across three consecutive testing days, then this was followed by a generalization phase with gradually increasing SF and gradually decreasing contrast. The orangutans were slowly acclimated to these stimuli because the more difficult stimuli resulted in more errors, less reinforcement, and increased agitation in the orangutans. This phase continued three times a week for several months before our final threshold testing; access was limited due to the zoo schedule and training sessions were sometimes prevented for practical reasons.

For our final threshold measurements, we used the staircase method of stimulus presentation (Cornsweet, 1962; García-Pérez, 2001). The seven contrast levels served as the steps of the staircase. The staircase began at the highest contrast (easiest step) and progressed through the contrast values to the lowest contrast (most difficult step). At the start of each staircase, a single trial was given at each contrast beginning at the easiest until the orangutan made the first error. Then we switched to a 2-down-1-up rule: the orangutan now had to complete two correct trials at each contrast step before progressing to the next more difficult contrast ("2 down"), and whenever an error occurred we immediately returned to the previous step ("1 up"). Simulations using this criterion track a threshold level of $71 \%$ correct responses (Garcia-Perez, 2001). Each change in direction in the staircase is referred to as a reversal. A staircase was continued until eight errors had been made, and thresholds were calculated by averaging stimulus values (log contrasts) at the last six reversals. On each of the three testing days, the orangutans completed four separate staircases, one for each 
of the four grating frequencies $(2,4,10$, and $15 \mathrm{c} / \mathrm{deg})$. The order of presentation of staircases was counterbalanced across testing days. Thresholds were averaged over the three days for each spatial frequency and CS at that SF (1/threshold) was calculated.

A quality check with three human observers was completed to verify the validity of the apparatus and stimuli; this was done using the same apparatus and stimuli, under lighting that matched the stimulus luminance used at the zoo. However, due to practical circumstances this was carried out in a laboratory setting where ambient lighting could be controlled and adjusted to match measurements made at the zoo.

\section{Results}

\section{Training Results}

Sekali reached the training criterion with the $0.5 \mathrm{c} / \mathrm{deg}$ stimuli after approximately six weeks of training three days per week. Ramai took much longer to learn the discrimination, meeting the criterion after 10 weeks. Interestingly, Ramai reached the criterion three training days after we switched her procedure from verbal feedback with a food reward, to a whistle-blast with a food reward. A whistle was never used for Sekali. Individual differences in motivation and social behavior were the reason for this change in procedure; we observed that Ramai was less motivated by social reinforcement, and easily distracted by a human voice and eye contact. In contrast, Sekali demonstrated high motivation in response to social reinforcement.

\section{Threshold Values Produced by Staircase Procedure}

A threshold was estimated from each staircase run by averaging the contrast values at the last six reversals of the staircase. Contrast sensitivity scores (reciprocal of threshold) for each orangutan on each test day at each test SF are shown in Table 3, along with their CS averaged across the three test sessions. $\mathrm{CS}$ was highest at $2 \mathrm{c} / \mathrm{deg}$ for both orangutans and declined gradually as SF increased. Figure 2 shows sample staircase data for Sekali at the lowest SF tested ( $2 \mathrm{c} / \mathrm{deg}$ ) and for Ramai at the highest SF (15 c/deg). These figures reveal two problems encountered using the staircase that led us to believe that the thresholds measured underestimate the orangutan's optimal performance, particularly at low SF. As can be seen in Figure 2A, despite extensive prior experience with these stimuli, some learning effect was evident with the staircase procedure in that more errors were made at the higher contrasts on the first run than on the later runs. This general pattern was displayed by both orangutans at the lower SF ( 2 and $4 \mathrm{c} / \mathrm{deg}$ ) and by Sekali at the higher frequencies as well. Secondly, it is clear that at $2 \mathrm{c} / \mathrm{deg}$ Sekali shows a ceiling level of performance with most trials at the most difficult stimulus level. In the final session, after two errors she made 23 consecutive correct responses to this stimulus; and there were several instances of runs of six or more consecutive correct responses at the lowest contrast step at SF of both 2 and $4 \mathrm{c} / \mathrm{deg}$. While not showing such a pronounced ceiling effect, Ramai also achieved runs of five consecutive correct responses for both of these lower SF. This indicates a serious limitation of the printed stimuli we used; the lowest contrast (0.03) we were able to produce was equivalent to a CS of only 33, whereas CS values of over 150 (contrast < 0.007) have been reported for humans. Thus from the staircase data alone we are not able to make a good estimate of CS at low SF. 
Adams et al. 213

Table 3

Contrast Sensitivity Averaged Over the Last Six Reversals from Three Testing Sessions

\begin{tabular}{|c|c|c|c|c|c|}
\hline & Testing day & $2 \mathrm{c} / \mathrm{deg}$ & $4 \mathrm{c} / \mathrm{deg}$ & $10 \mathrm{c} / \mathrm{deg}$ & $15 \mathrm{c} / \mathrm{deg}$ \\
\hline \multirow[t]{4}{*}{ Sekali } & Day 1 & 19.25 & 17.67 & 2.31 & 2.90 \\
\hline & Day 2 & 13.52 & 19.25 & 11.30 & 2.44 \\
\hline & Day 3 & $33.33^{\mathrm{a}}$ & 14.38 & 4.16 & 8.61 \\
\hline & Average CS & 22.03 & 17.10 & 5.92 & 4.65 \\
\hline \multirow[t]{4}{*}{ Ramai } & Day 1 & 14.77 & 5.85 & 2.71 & 1.39 \\
\hline & Day 2 & 16.23 & 11.98 & 1.73 & 1.53 \\
\hline & Day 3 & 17.67 & 14.72 & $2.24^{\mathrm{b}}$ & 1.44 \\
\hline & Average CS & 16.23 & 10.85 & 2.23 & 1.45 \\
\hline
\end{tabular}

${ }^{a}$ On Day 3 Sekali selected the target stimulus at the lowest contrast at $2 \mathrm{c} / \mathrm{deg}$ for 23 sequential trials so the trial was concluded after two warm-up errors (40 trials total).

${ }^{\mathrm{b}}$ On Day 3 Ramai only had four reversals despite eight errors due to a drop in affect. 

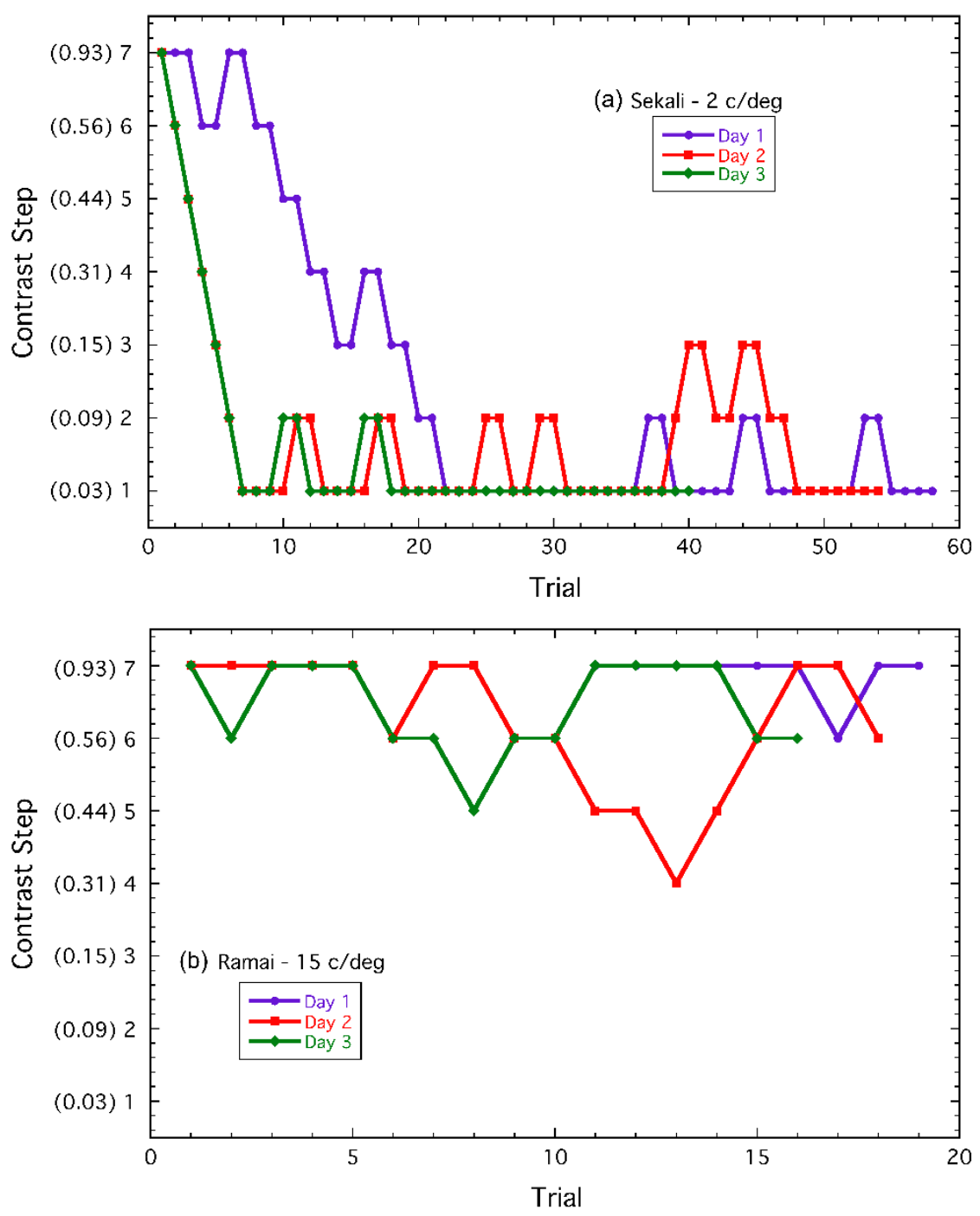

Figure 2. Examples of staircase progression for Sekali at $2 \mathrm{c} / \mathrm{deg}(\mathbf{A})$ and Ramai at $15 \mathrm{c} / \mathrm{deg}(\mathbf{B})$; trial number is plotted against contrast sensitivity step for three days. With successful performance, the orangutan progresses down the staircase from high contrast (0.93) to the lowest contrast (0.03) steps. Note that the contrast steps do not represent equal increments in contrast, and due to the number of trials, the scales are not the same.

At the highest SF tested (15 c/deg; see Figure 2B) Ramai's performance was close to chance, suggesting that we were able to approach the acuity limit with our available stimuli. Not apparent from the graph is the fact that approximately $50 \%$ of trials at the highest contrast $(0.93)$ were errors but, as we were at the top of the contrast scale, the staircase could not go further up. Sekali showed poor performance on the first day she was tested with the higher frequency patterns, and improved although performance was quite variable thereafter.

Thus, based on the staircase analysis alone, we can say only that the CSF of both orangutans peaked at $2 \mathrm{c} / \mathrm{deg}$ or below, and that at the high end, the orangutans were approaching or had reached the acuity limit $(\mathrm{CS}=1)$ by $15 \mathrm{c} / \mathrm{deg}$. Factors beyond our control precluded more extensive testing. Therefore, in order to optimize our threshold estimates, we have also used a second approach to analyzing the staircase data. 


\section{Analysis Using Percentage of Correct Responses}

Although under ideal conditions adaptive procedures like the staircase track thresholds efficiently, they provide little information about performance at superthreshold stimulus values. In order to take advantage of all the data collected, we also looked at cumulative scores (\% correct) on each contrast/SF combination tested. This allowed consideration of all trials $(\mathrm{N}=835)$, rather than just the reversal trials $(\mathrm{N}$ = 144). In order to estimate thresholds from this data we plotted the percent correct scores against log contrast for each SF for each orangutan, fit these data using a Quick function (Quick, 1974) and defined the threshold as the interpolated $75 \%$ correct value.

Contrast sensitivity values (1/threshold) derived from the staircase (red) and Quick function (blue) thresholds are plotted against SF in Figure 3. The average of the staircases (red) is derived from Table 3. In order to obtain an estimate of the function that would relate CS to SF over the full SF range, we adapted the model used by Wilson and Giese (1977) to fit human data for sustained stimuli: CSF $=A^{*} \omega * e^{-\omega / \sigma}$
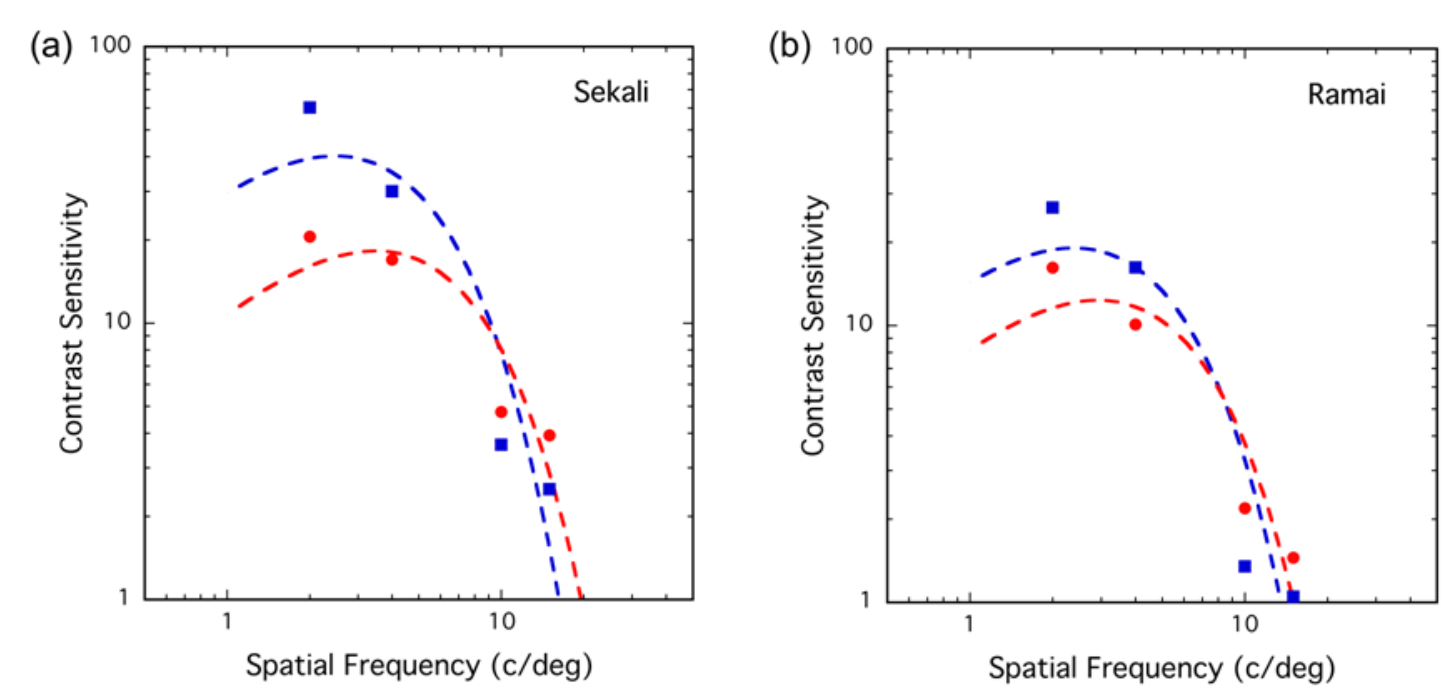

Figure 3. Contrast sensitivity function of the two orangutans: Sekali (A) and Ramai (B). The log scale of CS is plotted against log scale of spatial frequency $(2,4,10,15 \mathrm{c} / \mathrm{deg})$. The red line indicates the CSF derived from the staircase, and the blue line shows CS from the percent correct Quick fit.

Spatial frequency is denoted by $\omega$. The function has two free parameters: A is a gain factor determining the overall height of the function and $\sigma$ is the SF at which the function peaks. In Figure 3, the dashed red and blue lines are the best fits to the thresholds estimated from the staircase and Quick function analyses respectively for Sekali (Figure 3A) and Ramai (Figure 3B). For both orangutans, estimates of grating acuity $(\mathrm{CS}=1)$ are very similar using the two threshold estimate techniques (Sekali: $19.5 \& 16.2$ c/deg; Ramai: 15.0 \& $13.5 \mathrm{c} / \mathrm{deg}$ ). Although the Quick function measurements yielded higher CS at low SF, peak sensitivity was predicted in the same region $(2.4-3.5 \mathrm{c} / \mathrm{deg})$ for both orangutans and model fits. It should be noted that this model, which is based on human sustained data, has a low-frequency fall-off built into it. Based on our behavioral data alone, both orangutans showed greatest sensitivity at $2 \mathrm{c} / \mathrm{deg}$, so we cannot rule out the possibility that the shape of the underlying function peaks at or even below $2 \mathrm{c} / \mathrm{deg}$ in this species. Further testing with lower contrast stimuli at these SF would have provided more information about the curve; however, this was the lowest contrast that we could produce with the printer and we would have faced parallel difficulties displaying reliable contrast on a screen.

For the purpose of comparison, we used the same model to fit the CS data for four chimpanzees and one human published by Matsuno and Tomonaga (2006a; see Figure 4A); exact data values were generously provided to us by the authors. In their study, thresholds were measured over a range of five SF 
from 0.5 to $8 \mathrm{c} / \mathrm{deg}$. As can be seen in Figure 4A, the chimpanzee and human functions are a very good fit to the data, and peak sensitivity falls between 1 and $2 \mathrm{c} / \mathrm{deg}$ for all chimpanzees and for the human tested under comparable conditions. In Figure 4B we compare those data to the CSF functions (Quick thresholds) for the orangutans shown in blue. Here we have combined the data of three chimpanzees with similar data to produce an "average chimpanzee function." The fourth chimpanzee (C3 in Figure 4A) showed lower CS, possibly due to an ocular problem that was detected later (T. Matsuno personal communication Jan 28, 2016; Kaneko, Sakai, Miyabe-Nishiwaki, \& Tomonaga, 2013), so it was not included in this average. It is clear that while overall sensitivity was lower in the orangutans, peak sensitivity lies at the same SF and visual acuity, as estimated by extrapolating the function to $\mathrm{CS}=1$, is at least as high in the orangutans as in the chimpanzees in Matsuno and Tomonaga (2006a).
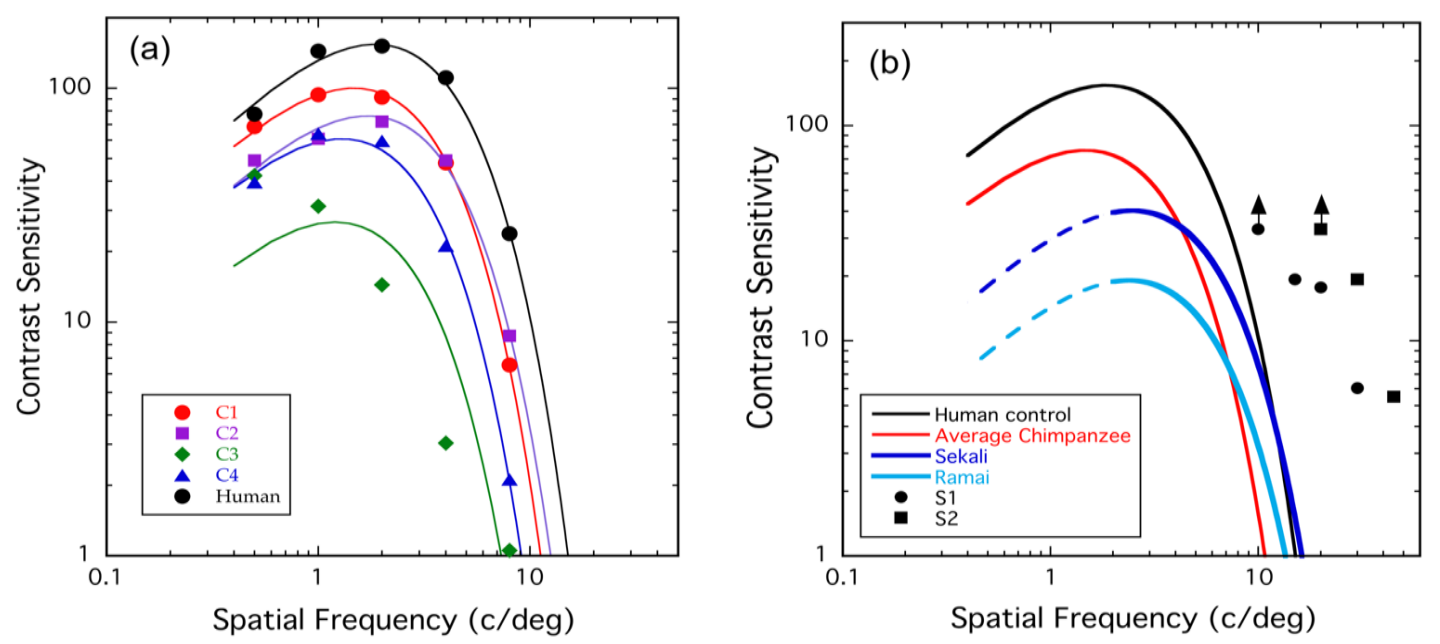

Figure 4. (A) CSF fits to the data of four chimpanzees and one human control generously provided by Matsuno and Tomonaga (2006a). Chimpanzee 3 was later found to have an arachnoid cyst in the visual area (Kaneko et al., 2013). (B) CSF for Sekali and Ramai fit as described in the text and displayed with the functions fit to the Matsuno \& Tomonaga results for comparison; For Sekali and Ramai solid lines are used for the portion of the curve derived with actual data and dashed lines for extrapolated data. Also shown are staircase-derived contrast thresholds for two human subjects tested with our procedures and stimuli. Data points with arrows indicate the highest SF at which their performance was $100 \%$ correct for our lowest contrast stimulus, indicating that their true threshold lies above the indicated point.

We also tested three human subjects ( 2 authors and 1 additional adult female). We used the same apparatus and stimuli as with the orangutans, illuminated by overhead lighting adjusted so that the stimulus luminance matched that of the zoo stimuli $\left(20 \mathrm{~cd} / \mathrm{m}^{2}\right)$. Procedures were identical to those reported above except food reinforcement was omitted. Two of the three humans performed perfectly at all SFs and contrasts at $57 \mathrm{~cm}$ and the third made errors only at $15 \mathrm{c} / \mathrm{deg}$ and the lowest contrast. Because thresholds could not be assessed under these conditions, two subjects were also tested at $114 \mathrm{~cm}$ and at $171 \mathrm{~cm}$, which increased the range of high SFs tested to include 20,30 and $45 \mathrm{c} / \mathrm{deg}$. Because the subjects had $100 \%$ correct performance for all SFs below $15 \mathrm{c} / \mathrm{deg}$ at all test distances, we could not estimate the location of the peak sensitivity or fit a meaningful function to the data. However, based only on thresholds at 20,30 and 45 $\mathrm{c} / \mathrm{deg}$, which are plotted in Figure $4 \mathrm{~b}$, it was clear the cutoff $(\mathrm{CS}=1)$ for one individual would fall between 30 and $45 \mathrm{c} / \mathrm{deg}$ whereas the other would fall above $45 \mathrm{c} / \mathrm{deg}$. This confirms that our procedures produce comparable high SF contrast thresholds to those produced in more traditional human psychophysical setups (Campbell \& Robson, 1968; De Valois et al., 1974). 


\section{Discussion}

The CSF as a description of the spatial sensitivity of the visual system is characterized by its overall height, the position of its peak along the spatial frequency axis, and its cutoff frequency, the SF beyond which the stimulus pattern is not resolvable even at maximum contrast $(\mathrm{CS}=1)$. In the present study, we have shown that the CSF of orangutans is generally similar to that of other species studied in terms of shape (Uhlrich et al., 1981), and its peak $(2-3 \mathrm{c} / \mathrm{deg})$ falls at the low end of the range reported for similar luminance conditions in primates (see Table 1). However, overall sensitivity is - at least under our test conditions - considerably lower than that reported for diurnal haplorhines tested in laboratory conditions (Table 1), and extrapolated cutoff spatial frequency (visual acuity) was found to be between 13 and $20 \mathrm{c} / \mathrm{deg}$ in the orangutans, again lower by a factor of three than the best estimates in humans and macaques (45 $60 \mathrm{c} / \mathrm{deg}$ ). It is possible that this indicates a marked divergence of orangutan spatial vision from that of other great apes, and given its very separate evolutionary history, its more arboreal lifestyle, and the extreme lack of existing documentation of the anatomical and physiological properties of its visual system, this possibility cannot be ruled out. However, it seems more likely that the differences can be explained more simply in terms of aspects of the test conditions. In humans and macaques, a wide range of stimulus and subject variables have been shown to affect both the overall level of sensitivity and the location of the peak of the CSF (e.g., luminance: De Valois et al., 1974; stimulus size: Campbell \& Robson, 1968; subject age: Arundale, 1978; Boothe et al., 1988).

\section{Comparison to Chimpanzees}

The only prior CSF measurement in a non-human great ape is the study of chimpanzees reported by Matsuno and Tomonaga (2006a). They collected data at five spatial frequencies between 0.5 and $8 \mathrm{c} / \mathrm{deg}$ using the PEST procedure, an adaptive staircase that generally requires fewer trials to estimate thresholds than the staircase used in the present study (Taylor \& Creelman, 1967). The function we used to fit our data also provided an excellent fit to the chimpanzee thresholds; the chimpanzees displayed greater sensitivity at the peak (average peak CS $=128$ ) than the orangutans, but the location of the peak was lower in the chimpanzees (average peak $=1.3 \mathrm{c} / \mathrm{deg}$ vs $2.8 \mathrm{c} / \mathrm{deg}$ ). Importantly, the extrapolated high frequency cut-offs in CS were also low, giving acuity estimates $(9-12 \mathrm{c} / \mathrm{deg})$, which fall below those of the orangutans, and far below the acuity measures reported in two chimpanzees by Spence (1934) using diffraction gratings (approximately 35 and $60 \mathrm{c} / \mathrm{deg}$ in the luminance range tested here), and in one chimpanzee tested by Matsuzawa (1990) using a letter matching test (letter acuity $=1.5$; equivalent to grating acuity of $45 \mathrm{c} / \mathrm{deg}$ ). Surprisingly, the single human subject in the Matsuno and Tomonaga (2006a) study showed a similar pattern of unexpectedly low peak location $(1.9 \mathrm{c} / \mathrm{deg})$ and cutoff SF $(15.1 \mathrm{c} / \mathrm{deg})$ even though the CS value at the peak (155) was in the low-normal human range. In contrast, human participants in our study showed performance at higher spatial frequencies that was much better than that of the orangutan subjects and predicted cutoff SFs in the normal human range of 35-50 c/deg. This suggests that the height and position of the CSF in the Matsuno and Tomonaga (2006a) study may have been affected by some aspect of their stimuli impacting human and chimpanzees alike, whereas in our study, the low thresholds are more likely due to differences in either motivation or testing procedure for the orangutans. A likely candidate limitation in the chimpanzee study is the limited size of the Gabor pattern stimuli. A study of macaques (Macaque nemestrina) by Kiorpes and Kiper (1996) with small targets and short stimulus exposures produced very similar CSF functions to those reported in the chimpanzees and in orangutans in the present study, despite high luminance, and testing procedure otherwise identical to those used in earlier studies from this group.

\section{Possible Contributors to the Lower CSF of the Orangutan}

At the lowest spatial frequency for which we gathered contrast thresholds $(2 \mathrm{c} / \mathrm{deg})$, our measurements undoubtedly underestimate the true abilities of the two orangutans because they both showed ceiling level performance at the lowest contrast stimulus we were able to produce, as did all of the human 
subjects tested in our apparatus. This was a serious limitation of our hard-copy stimuli as it allows us to say with certainty only that threshold contrast at $2 \mathrm{c} / \mathrm{deg}$ is considerably better than the lowest contrast we were able to present. However, at the higher frequencies, especially 10 and $15 \mathrm{c} / \mathrm{deg}$, the range of stimulus contrasts available was adequate to track threshold performance using our staircase procedure, and when we incorporated all test trials into our analysis to calculate $75 \%$ correct performance, thresholds changed very little at 10 and 15 cycles (see Figure 3). Overall luminance and stimulus size are not likely to account for the depressed performance as luminance was in the same range as those reported in Table 1, and our stimuli were large, and close to the animals. Viewing distance was quite constant as the orangutans moved their eyes right up to the cage mesh. In view of our own observations and details provided in the reports of other studies (Cowey \& Ellis, 1967; Spence \& Fulton, 1936; Tigges, 1963) we speculate that a combination of the distracting conditions in the zoo, task difficulty, and fluctuating motivation levels in combination with limitations in the amount of data we were able to collect within and across test sessions together account for the thresholds we obtained. The orangutans were rewarded with pieces of preferred food; however, unlike many earlier primate studies (e.g., Cowey \& Ellis, 1967), the orangutans were not food deprived. The orangutans were tested in their home enclosures so distractions included view of conspecifics and enrichment objects. Other reports of performance in orangutans reflect a similar failure to maintain focus and poor performance on simple discriminations (Davis \& Markowitz, 1978; Schrauf \& Call, 2009) and on list learning (Swartz et al., 2007). Other studies of Ramai and Sekali along with other Toronto zoo orangutans have shown a similar pattern to slow discrimination acquisition and maintenance performance of well under 100\% correct (Marsh et al., 2011), including studies on topics that we would expect to elicit more intrinsic interest such as picture recognition (Marsh \& MacDonald, 2008). It would be interesting in light of the present results to filter complex stimuli used in cognitive studies using the high frequency attenuation our current results suggest: this might reveal the aspects of the patterns most salient to orangutans.

The task we used in our study (vertical vs horizontal orientation discrimination), which has been used in other acuity measures (Ordy \& Samorajski, 1968; Treff, 1967; Veilleux \& Kirk, 2009), provides better experimental control than the more common stripes versus homogeneous field, but it is more difficult for animals to learn and maintain. Whereas the orangutans were extensively over-trained on a range of spatial frequencies and contrasts after reaching our learning criterion, they nevertheless showed variable performance across days on the staircase threshold assessments, and made mistakes on early trials. The staircase itself may also have provoked frustration in the animals, as easy trials occurred only at the beginning of a test. Spence and Fulton (1936) commented that one of his chimpanzees hated to make errors and would have temper tantrums or stop responding when the task became at all difficult, and the other made careless errors throughout leading to a much flatter frequency-of-seeing curve. Tigges (1963) similarly reported temper tantrums in a young orangutan when transferred to a more difficult version of a grey versus yellow color discrimination. Unfortunately, by the time we became aware of some of the shortcomings of the staircase procedure as we had implemented it, we were not able to improve the protocol due to lack of continuing access to the orangutans as they were involved in new studies. If such a study were repeated, we would recommend changing the testing in the following ways: 1) set a much longer reversal criterion - for example test until 12 reversals had occurred and average only the last 8,2 ) insert occasional much easier "reminder" trials into the staircase procedure to reduce frustration, and 3) run several additional days of staircase testing so that the first $1-2$ days could be treated as practice and not included in final threshold calculations. Even with these changes, we doubt that thresholds in the orangutans would have been improved to the level reported in laboratory testing of diurnal primates, as too many other factors can impact performance in the zoo setting.

\section{Future Questions and Conclusions}

The present study provides a first look at spatial vision in Sumatran orangutans. We hope that future studies will examine other visual abilities of orangutan, particularly visual motion and depth perception, as these are skills critical to arboreal survival. A comparison of CSF across primate species and ecological 
niches would also be a valuable next step. CSF has been measured in only a handful of primate species, the majority of which are habitat generalists, making ecological hypotheses difficult (Table 1). Future research could compare CSF across diverse primate species, in particular across diurnal/nocturnal niche, phylogenetic branches, and habitat. Comparisons across mammals, birds, and fish have found a similar shape of CSF despite differences in acuity and contrast sensitivity (Uhlrich et al., 1981), with birds showing high acuity but low contrast sensitivity (Ghim \& Hodos, 2006). It would be interesting to examine whether this trade-off is observed in primates. In particular, it would be useful to compare the CSF shape, acuity, and contrast sensitivity for species specialized to habitats at extremes of light (e.g., a tropical rainforest species that encounters only filtered light versus a species that lives exclusively in bright open savanna). Our results suggest that the orangutan may not have as sensitive spatial vision as expected from considerations of eye size, phylogeny, and niche (Veilleux \& Kirk, 2014). However, an answer to the question of whether the orangutan spatial vision is truly anomalous will await further behavioral studies, and anatomical assessment of the Nyquist limit of the foveal retinal mosaic and the pattern of connections between foveal cones and midget ganglion cells, the two main anatomical determinants of human visual acuity (Rossi \& Roorda, 2010; Williams, 1988). In the meantime, our findings provide a valuable picture of the range of visual stimuli that one can be confident will be effective as experimental stimuli and as environmental enrichment for the orangutan.

\section{Acknowledgements}

We would like to thank Sarah Ritvo for her assistance with data collection, Dr. Hugh R. Wilson for help with the CSF curve-fitting, Dr. T. Matsuno and Dr. M. Tomonaga for providing comparative data, and Linda Lillikas for assistance with graphics. We gratefully acknowledge funding support provided by a Natural Sciences and Engineering (NSERC) Discovery grant 7551 to F. E. Wilkinson. We also thank the staff at the Toronto Zoo for their assistance and continued support.

\section{References}

Arundale, K. (1978). An investigation into the variation of human contrast sensitivity with age and ocular pathology. British Journal of Ophthalmology, 62, 213 - 215.

Bard, K. A., Street, E. A., McCrary, C., \& Boothe, R. G. (1995). Development of visual acuity in infant chimpanzees. Infant Behavior and Development, 18, 225 - 232.

Becquet, C., \& Przeworski, M. (2007). A new approach to estimate parameters of speciation models with application to apes. Genome Research, 17, $1505-1519$.

Boothe, R. G., Kiorpes, L., Williams, R. A., \& Teller, D. Y. (1988). Operant measurements of contrast sensitivity in infant macaque monkeys during normal development. Vision Research, 28, 387 - 396.

Campbell, F. W., \& Robson, J. G. (1968). Application of Fourier analysis to the visibility of gratings. The Journal of Physiology, 197, $551-566$.

Chan, T. L., \& Grünert, U. (1998). Horizontal cell connections with short wavelength-sensitive cones in the retina: A comparison between New World and Old World primates. Journal of Comparative Neurology, 393, 196 209.

Cornsweet, T. N. (1962). The staircase-method in psychophysics. The American Journal of Psychology, $75,485-$ 491.

Cowey, A., \& Ellis, C. M. (1967). Visual acuity of rhesus and squirrel monkeys. Journal of Comparative and Physiological Psychology, 64, $80-84$.

Davis, R. R., \& Markowitz, H. (1978). Orangutan performance on a light-dark reversal discrimination in the zoo. Primates, 19, $755-759$.

De Valois, R. L., Morgan, H. C., \& Snodderly, D. M. (1974). Psychophysical studies of monkey vision-III. Spatial luminance contrast sensitivity tests of macaque and human observers. Vision Research, 14, $75-81$.

De Sousa, A. A., Sherwood, C. C., Mohlberg, H., Amunts, K., Schleicher, A., MacLeod, C. E., ...Zilles, K. (2010). Hominoid visual brain structure volumes and the position of the lunate sulcus. Journal of Human Evolution, $58,281-292$.

García-Pérez, M. A. (2001). Yes-no staircases with fixed step sizes: Psychometric properties and optimal setup. 
Optometry \& Vision Science, 78, $56-64$.

Ghim, M. M., \& Hodos, W. (2006). Spatial contrast sensitivity of birds. Journal of Comparative Physiology A, 5,523 $-534$.

Gilad, Y., Wiebe, V., Przeworski, M., Lancet, D., \& Pääbo, S. (2004). Loss of olfactory receptor genes coincides with the acquisition of full trichromatic vision in primates. PLoS Biol, 2, e5.

Goodman, M., Porter, C. A., Czelusniak, J., Page, S. L., Schneider, H., Shoshani, J., ...Groves, C. P. (1998). Toward a phylogenetic classification of primates based on DNA evidence complemented by fossil evidence. Molecular Phylogenetics and Evolution, 9, 585 - 598.

Hardus, M. E., Lameira, A. R., Zulfa, A., Atmoko, S. S. U., de Vries, H., \& Wich, S. A. (2012). Behavioral, ecological, and evolutionary aspects of meat-eating by Sumatran orangutans (Pongo abelii). International Journal of Primatology, 33, 287 - 304.

Hotta, G. (1905). Das auge der anthropoiden affen. Graefes Archiv fur Opthalmologie, 62, 250 - 274.

Jacobs, G. H. (1977). Visual capacities of the owl monkey (Aotus trivirgatus)—I. Spectral sensitivity and color vision. Vision Research, 17, $811-820$.

Jacobs, G. H. (1996). Primate photopigments and primate color vision. Proceedings of the National Academy of Sciences, 93, $577-581$.

Kaneko, T., Sakai, T., Miyabe-Nishiwaki, T., \& Tomonaga, M. (2013). A case of naturally occurring visual field loss in a chimpanzee with an arachnoid cyst. Neuropsychologia, 51, 2856-2862.

Kiorpes, L., \& Kiper, D. (1996). Development of contrast sensitivity across the visual field in macaque monkey (Macaca nemistrina). Vision Research, 36, 239 - 247.

Kirk, E. C. (2004). Comparative morphology of the eye in primates. The Anatomical Record Part A: Discoveries in Molecular, Cellular, and Evolutionary Biology, 281, 1095 - 1103.

Kirk, E. C. (2006). Effects of activity pattern on eye size and orbital aperture size in primates. Journal of Human Evolution, 51, $159-170$.

Langston, A., Casagrande, V. A., \& Fox, R. (1986). Spatial resolution of the Galago. Vision Research, 26,791 - 796.

Lesmes, L., Lu, Z.-L., Baek, J., \& Albright, T. (2010). Bayesian adaptive estimation of the contrast sensitivity function: the quick CSF method. Journal of Vision, 10, $1-21$.

Mailund, T., Dutheil, J. Y., Hobolth, A., Lunter, G., \& Schierup, M. H. (2011). Estimating divergence time and ancestral effective population size of Bornean and Sumatran orangutan subspecies using a coalescent hidden Markov model. PLoS Genetics, 7, e1001319.

Marsh, H. L., \& MacDonald, S. E. (2008). The use of perceptual features in categorization by orangutans (Pongo abelli). Animal Cognition, 11, $569-585$.

Marsh, H. L., Spetch, M. L., \& MacDonald, S. E. (2011). Strategies in landmark use by orangutans and human children. Animal Cognition, 14, 487 - 502.

Matsui, A., Go, Y., \& Niimura, Y. (2010). Degeneration of olfactory receptor gene repertories in primates: No direct link to full trichromatic vision. Molecular Biology and Evolution, 27, 1192 - 1200.

Matsuno, T., \& Fujita, K. (2009). A comparative psychophysical approach to visual perception in primates. Primates, $50,121-130$.

Matsuno, T., \& Tomonaga, M. (2006a). Measurement of contrast thresholds of chimpanzees using a parameter estimation by sequential testing (PEST) procedure. The Japanese Journal of Psychonomic Science, 25, 115 $-116$.

Matsuno, T., \& Tomonaga, M. (2006b). Visual search for moving and stationary items in chimpanzees (Pan troglodytes) and humans (Homo sapiens). Behavioural Brain Research, 172, 219 - 232.

Matsuno, T., \& Tomonaga, M. (2008). Temporal characteristics of visibility in chimpanzees (Pan troglodytes) and humans (Homo sapiens) assessed by a visual-masking paradigm. Perception, 37, 1258 - 1268.

Matsuzawa, T. (1990). Form perception and visual acuity in a chimpanzee. Folia Primatologica, 55, 24 - 32.

Merigan, W. H. (1976). The contrast sensitivity of the squirrel monkey (Saimiri sciureus). Vision Research, 16, 375 $-379$.

Miles, H. (1990). The cognitive foundations for reference in a signing orangutan. In S. T. Parker \& K. R. Gibson (Eds.), "Language" and intelligence in monkeys and apes: Comparative developmental perspectives (pp. 511 - 539). New York, NY: Cambridge University Press.

Mitchell, D. E., Giffin, F., Wilkinson, F., Anderson, P., \& Smith, M. L. (1976). Visual resolution in young kittens. Vision Research, 16, 363-366.

Montiani-Ferreira, F., Lima, L., Bacellar, M., D’Otaviano Vilani, R. G., Fedullo, J. D., \& Lange, R. R. (2010). Case report: Bilateral phacoemulsification in an orangutan (Pongo pygmaeus). Veterinary Ophthalmology, 13, 91 -99 . 
O'Keefe, L. P., \& Movshon, J. A. (1998). Processing of first-and second-order motion signals by neurons in area MT of the macaque monkey. Visual Neuroscience, 15, $305-317$.

Ordy, J., \& Samorajski, T. (1968). Visual acuity and ERG-CFF in relation to the morphological organization of the retina among diurnal and nocturnal primates. Vision Research, 8, $1205-1225$.

Owsley, C., \& Sloane, M. (1987). Contrast sensitivity, acuity, and the perception of 'real-world' targets. British Journal of Ophthalmology, 71, $791-796$.

Preuss, T. M., Qi, H., \& Kaas, J. H. (1999). Distinctive compartmental organization of human primary visual cortex. Proceedings of the National Academy of Sciences, 96, 11601 - 11606.

Preuss, T. M., \& Coleman, G. Q. (2002). Human-specific organization of primary visual cortex: Alternating compartments of dense Cat-301 and calbindin immunoreactivity in layer 4A. Cerebral Cortex, 12, 671-691.

Quick Jr, R. F. (1974). A vector-magnitude model of contrast detection. Kybernetik, 16, 65 - 67.

Rijksen, H. D. (1978). A field study on Sumatran orangutans (Pongo pygmaeus abelii, Lesson 1827): Ecology, behaviour and conservation. Wageningen, Netherlands: H. Veenman.

Ross, J. E., Clarke, D. D., \& Bron, A. J. (1985). Effect of age on contrast sensitivity function: Uniocular and binocular findings. British Journal of Ophthalmology, 69, $51-56$.

Rossi, E. A., \& Roorda, A. (2010). The relationship between visual resolution and cone spacing in the human fovea. Nature Neuroscience, 13, 156 - 157.

Schmitt, D. (2010). Primate locomotor evolution: Biomechanical studies of primate locomotion and their implications for understanding primate neuroethology. In M. L. Platt \& A. A. Ghazanfar, (Eds.), Primate neuroethology (pp. 31 - 63). Oxford, UK: Oxford University Press.

Schrauf, C., \& Call, J. (2009). Great apes' performance in discriminating weight and achromatic color. Animal Cognition, 12, $567-574$.

Semendeferi, K., Teffer, K., Buxhoeveden, D. P., Park, M. S., Bludau, S., Amunts, K., ...Buckwalter, J. (2011). Spatial organization of neurons in the frontal pole sets humans apart from great apes. Cerebral Cortex, 21, $1485-$ 1497.

Singleton, I., Wich, S.A., Nowak, M. \& Usher, G. (2016). Pongo abelii. (errata version published in 2016). The IUCN Red List of Threatened Species. Version 2017-1. Retrieved from http://iucnredlist.org.

Spence, K. W. (1934). Visual acuity and its relation to brightness in chimpanzee and man. Journal of Comparative Psychology, 18, $331-361$.

Spence, K. W., \& Fulton, J. F. (1936). The effects of occipital lobectomy on vision in chimpanzee. Brain: A Journal of Neurology, 59, $35-50$.

Swartz, K. B., Himmanen, S. A., \& Shumaker, R. W. (2007). Response strategies in list learning by orangutans (Pongo pygmaeus $\times$ P. abelii). Journal of Comparative Psychology, 121, $260-269$.

Taylor, M., \& Creelman, C. D. (1967). PEST: Efficient estimates on probability functions. The Journal of the Acoustical Society of America, 41, 782 - 787.

Tigges, J. (1963). On color vision in gibbon and orang-utan. Folia Primatologica, 1, 188 - 198.

Treff, H. A. (1967). Tiefensehscharfe und sehscharfe beim galago (Galago senegalensis). Zeitschrift fur vergleichende Physiologie, 54, 26 - 57.

Uhlrich, D., Essock, E., \& Lehmkuhle, S. (1981). Cross-species correspondence of spatial contrast sensitivity functions. Behavioural Brain Research, 2, 291 - 299.

van Schaik, C. P. (1999). The socioecology of fission-fusion sociality in orangutans. Primates, 40, 69 - 86.

van Schaik, C. (2004). Among orangutans: Red apes and the rise of human culture. Cambridge, MA \& London, UK: Harvard University Press.

Veilleux, C. C., \& Kirk, E. C. (2009). Visual acuity in the cathemeral strepsirrhine Eulemur macaco flavifrons. American Journal of Primatology, 71, $343-352$.

Veilleux, C., \& Kirk, E. (2014). Visual acuity in mammals: Effects of eye size and ecology. Brain Behaviour Evolution, 83, $43-53$.

Vonk, J. (2014). Quantity matching by an orangutan (Pongo abelii). Animal Cognition, 17, 297 - 306.

Vonk, J., \& MacDonald, S. E. (2004). Levels of abstraction in orangutan (Pongo abelii) categorization. Journal of Comparative Psychology, 118, 3 - 13.

Waitt, C., \& Buchanan-Smith, H. M. (2006). Perceptual considerations in the use of colored photographic and video stimuli to study nonhuman primate behavior. American Journal of Primatology, 68, 1054 - 1067.

Weinstein, B., \& Grether, W. F. (1940). A comparison of visual acuity in the rhesus monkey and man. Journal of Comparative Psychology, 30, $187-195$.

Wich, S. A., Utami-Atmoko, S. S., Setia, T. M., Rijksen, H. D., Schürmann, C., Van Hooff, J. A. R. A. M., \& van Schaik, C. P. (2004). Life history of wild Sumatran orangutans (Pongo abelii). Journal of Human Evolution, 
$47,385-398$.

Wilkinson, F. (1984). The development of visual acuity in the Mongolian gerbil (Meriones unguiculatus). Behavioural Brain Research, 13, 83 - 94.

Williams, D. R. (1988). Topography of the foveal cone mosaic in the living human eye. Vision Research, 28, 433 454.

Williams, R. A., Boothe, R. G., Kiorpes, L., \& Teller, D. Y. (1981). Oblique effects in normally reared monkeys (Macaca nemestrina): Meridional variations in contrast sensitivity measured with operant techniques. Vision Research, 21, $1253-1266$.

Wilson, H. R., \& Giese, S. C. (1977). Threshold visibility of frequency gradient patterns. Vision Research, 17, 1177 -1190 .

Zilles, K. A. R. L., \& Rehkämper, G. (1988). The brain, with special reference to the telencephalon. In J. H. Schwartz (Ed.), Orang-utan biology (pp. 157 - 176). Oxford, UK: Oxford University Press. 